\title{
How to Diagnose Exercise Induced Asthma?
}

Zarqa Ali

Author's Affiliation:

Copenhagen University, Denmark

* Corresponding Author;

Saxogade 106,1,-64

1662 Copenhagen V

Denmark

E-mail: zarqa_ali@hotmail.com

Received: Jan 25, 2011

Accepted: Apr 15, 2011

Key Words: Bronchospasm; Asthma, Exercise-Induced; Diagnosis; Athletes; Exercise

\begin{abstract}
Exercise induced asthma (EIA) is a transient increase in airway resistance after intensive exercise and can be measured as a decline in forced expiratory volume in one second (FEV1). The condition is due to increased training load and inhalation of cold and dry air. Several studies have shown that eucapnic voluntary hyperpnea challenge test (EVH test) is a very sensitive and specific diagnostic method. EVH test develops EIA by hyperventilation of dry gas and the test achieves the same airway obstruction as training in cold and dry air. The test is better than the previously used methacholine challenge test.
\end{abstract}

Asian Journal of Sports Medicine, Volume 2 (Number 2), June 2011, Pages: 63-67

\section{INTRODUCTION}

$\mathrm{E}$ xercise induced asthma (EIA) is a temporary increase in airway resistance after intensive sport and the airway obstruction can objectively be measured as a decline in Forced Expiratory Volume in One Second $\left(\mathrm{FEV}_{1}\right)$. Increased training load and the impact of the surroundings are responsible for EIA ${ }^{[1]}$. The prevalence of EIA is high among elite athletes, especially among athletes who do outdoor sport activities. The temperature and air humidity affects the stress level in the airway. Hence, the prevalence of EIA is higher among winter athletes compared with summer athletes. Cold and dry winter air is more harmful to the respiratory tract compared with hot and humid air in the summer ${ }^{[2]}$. The frequency of EIA during the Olympic Games (OG) has been gradually increasing in recent years, which has coursed concern at the International Olympic Committee-Medical Commission (IOC-MC).

The high frequency has resulted in greater use of bronchial dilators during OG. In the last couple of years, the participants could obtain permission to use bronchial dilators during the Olympics on the basis of self-reported symptoms. This resulted in an increase in the intake of medicine. To avoid doping and misuse of these drugs as performance enhancing drugs, IOC-MC require now documented evidence before therapeutic administration of these medications to the participants during $\mathrm{OG}^{[3,4]}$.

This issue is still essential, although there currently is no evidence indicating that bronchial dilators may encourage athletes' performance. Accordingly, currently available diagnostic methods for diagnosing EIA will be explained in this manuscript.

\section{METHOD}

A structured literature search was carried out in the PubMed database in keywords category with the following keywords: (Asthma or exercise-induced 
asthma or bronchoconstriction or bronchospasm) AND (exercise or activity or athlete). These keywords were used alone and in combination with screening and diagnosis. The search resulted in 67 published articles, relevant articles were reviewed and new related articles were included. All types of articles were searched and read, but our primary focus was on original articles. Although EIA has been studied in children and adults in numbers of previous articles, only studies which assessed EIA in athletes were enrolled. Our exclusion criteria were articles with focus on EIA in children and adults, while our inclusion criteria were articles focusing on EIA in athletes.

\section{RESULTS}

Finally 17 articles were enrolled in this study. Two articles were enrolled in direct diagnostic method and 13 in indirect diagnostic method.

\section{Diagnostic Methods:}

Several studies have shown that EIA is often misdiagnosed by both over and under diagnosis ${ }^{[5]}$. Which diagnostic methods are specific and sensitive for diagnosis of EIA is therefore an essential question.

EIA can be diagnosed by several methods. However, there is a large variation in their quality and sensitivity, and their prices are rarely comparable.

Diagnosis methods can generally be divided into two categories, direct and indirect tests. Direct test is a pharmacological test and induces a direct contraction of the smooth muscle in the airways by using medications. Indirect test induces contraction by mimicking the event on the sport field.

\section{Direct test:}

Methacholine is a drug which is used to measure the sensitivity of airways by causing a transient narrowing of the airways. It acts directly on acetylcholine receptors on the smooth muscle in the airways, resulting in an airway narrowing by contraction of muscles. Methacholine challenge test is a direct test and is also used in the clinic to diagnose chronic asthma. The test is carried out in a laboratory, where athletes are exposed to stepwise elevating doses of methacholine in the inspiration air, which results in bronchial contraction. This is measured by a pulmonary function test (PFT) after administration of each dose. The test is considered positive if the decline in $\mathrm{FEV}_{1}$ is greater than $20 \%$ from baseline value $\left(\mathrm{FEV}_{1}\right.$ measurements at rest). The fall in $\mathrm{FEV}_{1}$ must be presented with a methacholine concentration of less than or equal to $8 \mathrm{mg} / \mathrm{ml}^{[6,7]}$. The test is useful in the clinic to diagnose chronic asthma, but has a low sensitivity for the screening of EIA. The benefits of the test are that it is easily accessible, reproducible, not time-consuming, inexpensive and independent of special equipment such as treadmill and cycle ergometer. The disadvantages are that the test is laboratory-dependent and does not measure the outdoor environments stress which the athletes are exposed to during the exercise ${ }^{[6]}$.

\section{Indirect test:}

The indirect tests imitate the event on the sports field and reproduce EIA in the same way that sport would do under normal circumstances. The indirect tests include physical tests, eucapnic voluntary hyperpnea challenge test $(\mathrm{EVH})$ and osmotic challenge test.

There are two types of exercise tests; laboratory exercise challenge tests and field exercise challenge tests.

The laboratory exercise challenge tests are performed in a laboratory on a cycle ergometer or treadmill. Two different protocols can be used on the cycle ergometer. The first protocol is a single load, in which the subject exercises at an intensity of $45 \%$ to $60 \%$ of the predicted maximum voluntary ventilation (MVV) for 6 to 8 minutes. The second protocol is an increasing protocol, in which the subject begins with an intensity of $60 \%$ of the final load in the first minute, then $75 \%$ in the second minute, $90 \%$ in the third minute and $100 \%$ in the fourth minute. Once the target level is reached, the workload is held for 4 minutes ${ }^{[8]}$. The standardized treadmill protocol recommends a speed and grade to produce 4 to 6 minutes at near maximum heart rate, with a total exercise duration of 6 to 8 minutes. During the first 2 to 3 minutes, the 
treadmill speed and grade are rapidly increased until the subject achieves a heart rate of $80 \%$ to $90 \%$ of the maximum predicted. PFT is measured with regular intervals for up to 30 mins following completion of either challenge ${ }^{[6,9,10]}$. The test is considered positive if the fall in $\mathrm{FEV}_{1}$ is greater than $15 \%$ from baseline value. This method is specific for EIA, but it is not sport specific. The disadvantage is that the test is not performed in the environment, in which exercise is usually performed ${ }^{[6]}$.

Field exercise challenge test is performed in the sport, in which athletes are normally engaged. The athletes exercise in 4 to 6 minutes at $85 \%$ of their maximum heart rate, PFT is then measured regularly for up to $30 \mathrm{~min}$ after completion of the exercise. The fall in $\mathrm{FEV}_{1}$ must be greater than $15 \%$ before the test can be judged positive. This test is sport specific and specific for EIA, in which environmental conditions of temperature and humidity have also been considered $^{[6,11,12]}$.

The EVH test is an indirect challenge test, which reproduces EIA by hyperventilation of dry gas containing $5 \% \mathrm{CO}_{2}, 21 \% \mathrm{O}_{2}$ and balanced $\mathrm{N}_{2}$. These concentrations are safe and stimulate ventilation. The normal end-tidal $\mathrm{CO}_{2}$ level is held through the test. Inhalation of the gas dries the liquid on the airway surface, resulting in an increased osmolarity. This activates mast cells to release inflammatory mediators, whereby airway narrowing occurs, similar to exercise.

Two different protocols can be used, requiring different levels of MVV, which is calculated as a resting $\mathrm{FEV}_{1}$ multiplied by $35^{[6]}$.

The first protocol is a stepped protocol and is used for medium or serious EIA. The ventilation rate increases over three steps:

Step 1: 3 minutes at $30 \% \mathrm{MVV}$

Step 2: 3 minutes at $60 \% \mathrm{MVV}$

Step 3: 3 minutes at $90 \% \mathrm{MVV}$

PFT is measured after 1, 3, 5 and 7 minutes following completion of each step.

The second protocol is a single-step protocol and is used for mild EIA. The subject exercises with a ventilation of $85 \%$ of MVV for 6 minutes. PFT is measured for up to 15 minutes after the activity ${ }^{[6]}$. The fall in $\mathrm{FEV}_{1}$ must be greater than $10 \%$ to judge the test as positive. The test has a high specificity for EIA, is inexpensive and laboratory dependent. Furthermore, environmental stress is considered when the dry air is inhaled $^{[3,5,13]}$. EVH test is very sensitive, and the test achieves the same airway obstruction as exercise in dry and cold air ${ }^{[14]}$.

Numerous studies have shown that EVH test results in the diagnosis of most numbers of athletes compared with other tests, which give many false negative diagnoses. This confirms the EVH test's high sensitivity and is therefore recommended by the IOC$\mathrm{MC}^{[5,13,15]}$

Osmotic challenge test induces EIA by administrating elevating doses of dry powder mannitol or hypertonic saline to the inhalation air. PFT is measured following each administration. Hypertonic saline promotes hyperosmolarity and hypertonicity in the airways in the same way that exercise would do under normal circumstances. Mast cells degranulate and inflammatory mediators are released, which induce contraction of the bronchial musculature and promote airway narrowing. The test is effective in the same way as the EVH test and exercise, but it is more economical and easier to perform.

Mannitol is a naturally sugar alcohol in most vegetables. Mannitol facilitates contraction of smooth muscle in the airways by promoting the release of inflammatory mediators. The test is practical, not timeconsuming and independent of special equipment like cycle ergometer, treadmill or dry air source. The test is considered positive if the fall in $\mathrm{FEV}_{1}$ is greater than $15 \%$. The test is safe, inexpensive, sensitive and highly specific for $\mathrm{EIA}^{[3,16,17]}$.

\section{DISCUSSION}

Many different diagnostic methods have been used over the years to diagnose EIA, but the IOC-MC now recommends EVH test as the most reliable one. EVH test will in future be used during the Olympics before administration of bronchial dilators. EVH test imitates the event on the sports field and achieves the same 
reduction in lung function, which is achieved under real circumstances during outdoor exercise ${ }^{[14]}$. The inhalation of dry gas imitates the humidity and hyperventilation imitates the increased ventilation during exercise. Although the sport in which the athletes are daily engaged is not considered, the test manages to give the same drop in $\mathrm{FEV}_{1}^{[1]}$. EVH test is currently available only in specialized centers. As a result, the test is not widespread yet.

Methacholine test has widely been used through the years and is also appropriate for diagnosis of chronic asthma, but is neither sensitive nor specific for EIA ${ }^{[6]}$. There are many factors that affect EIA which are being ignored by the test such as temperature and humidity of the breathing air and the level of exercise. In spite of its limitations, methacholine test is very widespread due to its reproducibility, low cost and popularity in the clinic. Laboratory exercise challenge test considers more factors than methacholine test, but the disadvantage of this test is that it can only be performed on a treadmill or cycle ergometer. The test is not sport specific ${ }^{[6]}$. For instance, swimmers may not be able to perform their maximum on a cycle ergometer, which on the other hand is possible by swimming. Athletes will therefore rarely reach their maximum ventilation when performing the test. Another disadvantage of the test is that the competition element is not considered. Competition in sport is a motivating factor for participants, which contributes to increase in participants' performance. The participants rarely achieve enough ventilation on a treadmill or cycle ergometer which is needed to induce EIA. Field exercise challenge test is more realistic and considers more factors than laboratory exercise challenge tests. Although it seems like an optimal test, the test is difficult to perform in reality and is very timeconsuming. Standardization is very difficult in both terms of environmental stress, air temperature and humidity, and the cardiovascular workload. It is also difficult to compare the test results across different sports due to the lack of standardization.

The osmotic challenge test is both sensitive and specific, and causes a reduction in $\mathrm{FEV}_{1}$ much faster than any other procedures such as hyperventilation or exercise that can take up to 20 minutes. This test is recommended and mostly used.

Future studies should therefore be performed to compare the sensitivity of EVH test and and the osmotic challenge test to examine whether the osmotic challenge test can replace the EVH test. Evidence suggests that the osmotic challenge test in the future will also be useful to diagnose EIA.

\section{REFERENCES}

1. Wilber RL, Rundell KW, Szmedra L, et al. Incidence of exercise-induced bronchospasm in Olympic winter sport athletes. Med Sci Sports Exerc 2000;32:732-7.

2. Rundell KW, Jenkinson DM. Exercise-induced bronchospasm in the elite athlete. Sports Med 2002;32:583-600.

3. Rundell KW, Slee JB. Exercise and other indirect challenges to demonstrate asthma or exercise-induced bronchoconstriction in athletes. J Allergy Clin Immunol 2008;122:238-46.

4. Weinberger M. Long-acting beta-agonists and exercise. J Allergy Clin Immunol 2008;122:251-3.

5. Holzer K, Anderson SD, Douglass J. Exercise in elite summer athletes: Challenges for diagnosis. J Allergy Clin Immunol 2002; 110:374-80.

6. Holzer K, Brukner P. Screening of athletes for exercise-induced bronchoconstriction. Clin J Sport Med 2004;14:134-8.

7. Naranjo Orellana J, Centeno Prada RA, Carranza Márquez MD. Use of beta2 agonists in sport: are the present criteria right? Br J Sports Med 2006;40:363-6.

8. Holzer K, Brukner P. Screening of athletes for exercise-induced bronchoconstriction. Clin J Sport Med 2004;14:134-8.

9. Rundell KW, Wilber RL, Szmedra L, et al. Exercise-induced asthma screening of elite athletes: field versus laboratory exercise challenge. Med Sci Sports Exerc 2000;32:309-16. 
10. Verges S, Flore P, Laplaud D, et al. Laboratory running test vs. field roller skiing test in cross-country skiers: a longitudinal study. Int J Sports Med 2006;27:307-13.

11. Rundell KW, Anderson SD, Spiering BA, et al. Field exercise vs laboratory eucapnic voluntary hyperventilation to identify airway hyperresponsiveness in elite cold weather athletes. Chest 2004;125:909-15.

12. Stensrud T, Mykland KV, Gabrielsen K, et al. Bronchial hyperresponsiveness in skiers: field test versus methacholine provocation? Med Sci Sports Exerc 2007;39:1681-6.

13. Dickinson JW, Whyte GP, McConnell AK, et al. Screening elite winter athletes for exercise induced asthma: a comparison of three challenge methods. Br J Sports Med 2006;40:179-82.

14. Rundell KW, Spiering BA, Baumann JM, et al. Effects of montelukast on airway narrowing from eucapnic voluntary hyperventilation and cold air exercise. Br J Sports Med 2005;39:232-6.

15. Pohlig C. Exercise-induced bronchospasm: coding and billing for physician services. Chest 2009;135:210-4.

16. Choi IS, Chung SW, Koh YI, et al. Airway hyperresponsiveness to hypertonic saline as a predictive index of exercise-induced bronchoconstriction. Korean J Intern Med 2005;20:284-9.

17. Anderson SD, Charlton B, Weiler JM, et al. Comparison of mannitol and methacholine to predict exercise-induced bronchoconstriction and a clinical diagnosis of asthma. Respir Res 2009;10:4. 